\title{
Static Single-Arm Force Generation with Kinematic Constraints
}

\author{
Peng Pan Kevin M. Lynch Michael A.Peshkin J. Edward Colgate \\ Mechanical Engineering Department \\ Northwestern University, Evanston, IL 60208 \\ Email: panpeng \{kmlynch,peshkin,colgate\} @ northwestern.edu
}

\begin{abstract}
This study investigates natural single-arm interaction with kinematic constraints. Smooth, frictionless, kinematic constraints reduce the degrees-of-freedom of motion at the hand, but add force freedoms. These force freedoms allow the hand to push and pull against the constraints with no effect on the task. Understanding how subjects take advantage of kinematic constraints will be useful in designing constraint surfaces for assisted manipulation.

This paper reports the results of an experiment studying how subjects make use of the presence of a kinematic constraint in a static planar single-arm task. Subjects are asked to hold a handle that is free to slide on a linear rail, and to apply a force tangent to the rail to resist a pulling force. Thus the goal of the task is to hold the handle stationary. Subjects are also free to apply any force normal to the rail, as these forces have no effect on the task. This freedom does not exist without a kinematic constraint.

We find that subjects make use of the force freedom by applying significant forces against the constraint in a consistent and constraint-configuration-dependent fashion. We show that the constraint forces can be predicted by a convex, scale-invariant objective function on the hand force space. The level curves of this objective function can be found directly from the experimental data without any biomechanical modeling.
\end{abstract}

\section{INTRODUCTION}

We are investigating the use of passive robotic guides to assist a human in manipulating a load from one configuration to another. A guide acts as a workless, frictionless constraint surface that confines the load to a submanifold of its configuration space. A robot implementing a passive guide does not amplify human muscle power, but simply redirects the momentum of the load without affecting the energy. Our ultimate goal is to design and control assist robots to make manipulation of heavy loads faster, more comfortable, and less likely to result in work-related musculoskeletal disorders (WMSD's). We believe the more kinematic constraints the better in manipulation of a load, provided the constraints are consistent with the task and are the "right" constraints for the user. Understanding what "right" means is one motivation for this work.

Our focus is on manipulation of a load with $n$ degreesof-freedom before constraints are taken into consideration. For example, a rigid-body load has $n=6$, described by the body's x-y-z-roll-pitch-yaw coordinates. We assume the load is subject to $m$ independent, holonomic, equality configuration constraints. If $\mathbf{q}$ is the configuration of the body, these constraints can be expressed as $\mathbf{c}(\mathbf{q})=\mathbf{0}, \quad \mathbf{c}(\mathbf{q})=$ $\left(c_{1}(\mathbf{q}), \ldots, c_{m}(\mathbf{q})\right)$. The constraints are frictionless, so any generalized reaction force $\mathbf{f}_{r}$ (including forces and torques) acting on the load due to the constraints must be normal to the constraints, i.e., of the form

$$
\mathbf{f}_{r}=\sum_{i=1}^{m} w_{i} \frac{\partial c_{i}(\mathbf{q})}{\partial \mathbf{q}}, \quad w_{i} \in \mathbb{R} .
$$

A typical task is to move the load from one point of the constrained $(n-m)$-dimensional space to another. To accomplish this task, the human applies a generalized force $\mathbf{f}_{h}$ to the load. This force can be decomposed into orthogonal components (with respect to the kinetic energy metric), $\mathbf{f}_{h}=$ $\mathbf{f}_{n}+\mathbf{f}_{t}$, where $\mathbf{f}_{n} \in \mathcal{F}_{n}(\mathbf{q})$ and $\mathbf{f}_{t} \in \mathcal{F}_{t}(\mathbf{q}) . \mathcal{F}_{n}(\mathbf{q})$ is the $m$ dimensional space of generalized forces that do no work on the load at $\mathbf{q}$ (i.e., normal to the constraints), and $\mathcal{F}_{t}(\mathbf{q})$ is the $(n-m)$-dimensional set of generalized forces along the free motion directions at $\mathbf{q}$ (i.e., tangent to the constraints). Of the force $\mathbf{f}_{h}$, only the portion $\mathbf{f}_{t}$ contributes to solving the task; $\mathbf{f}_{n}$ sums with $\mathbf{f}_{r} \in \mathcal{F}_{n}(q)$ to ensure that the constraints are maintained, regardless of the human's choice of $\mathbf{f}_{n}$. ${ }^{1}$

Thus a typical task specification constrains the initial and final states of the load, but leaves the following freedoms:

1) Load trajectory in the free space. The human has freedom in choosing a path (and speed along the path) for the load in the $(n-m)$-dimensional free space to meet the terminal constraints. We will call these "motion freedoms."

2) Human body trajectory. A trajectory specification for the load implies constraints on the the trajectory of the human, typically the hand(s) manipulating the load. Due to redundancy, however, the human's joint trajectories may not be uniquely specified by the load trajectory.

3) Constraint forces. At each instant along the trajectory, the human can apply any generalized force in the $m$ dimensional space $\mathcal{F}_{n}(\mathbf{q})$ with no effect on the trajectory. We will call these "force freedoms."

4) Muscle load sharing. Given a specification of the freedoms (1-3) above, the joint torques are uniquely specified. However, the sharing of these joint torques across different muscle groups is not unique.

We are interested in how human subjects naturally make use of these freedoms in practiced constrained motion.

${ }^{1}$ In this discussion we ignored the possible role of gravitational forces, which generally will consist of components in both $\mathcal{F}_{n}(q)$ and $\mathcal{F}_{t}(q)$. 


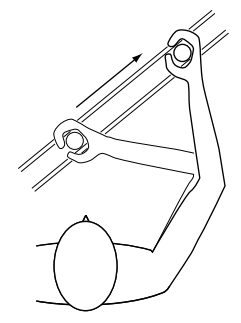

Fig. 1. An example planar single-arm reaching task, constrained by a linear guide rail.

We begin our investigation into natural human interaction with kinematic constraints by studying manipulation with a single arm in a horizontal plane. By immobilizing the wrist, we can treat the arm as a planar $2 \mathrm{R}$ manipulator (shoulder and elbow). The hand carries a load from one point to another in the plane, constrained by a guide rail to move along a fixed path (Figure 1). In our first set of experiments we study natural static interaction with a constraint. The subject holds a handle attached to a smooth linear rail and is asked to hold the handle stationary while the handle is pulled with different forces tangential to the rail. We are interested in the forces applied by the subject normal to the rail, which are not necessary for the task but may simplify the task for the subject. Thus this paper addresses the force freedoms (3) above.

\section{A. Key Questions}

The key questions and answers in this paper are:

1) Do subjects apply forces against the constraints? Such forces have no effect on the task. Despite the fact that the application of constraint forces increases the total force magnitude at the hand, the data show that subjects consistently apply forces against the constraint, in a constraint-configuration-dependent manner.

2) Do subjects' constraint forces scale proportionally with forces applied in the free direction? The answer appears to be yes, at least for relatively low applied forces.

3) Can the constraint forces be explained by an objective function model governing forces applied at the hand? The answer appears to be yes. This objective function should be viewed as an explanation and predictor for the data; it does not require that we commit to a particular interpretation of it (e.g., that subjects are attempting to minimize "effort").

4) Is it possible, from the data alone, to reconstruct level curves of the objective function implicit in the subject's interaction with the constraint? The answer is yes, and this reconstruction is independent of any strong assumption on the form of the objective function or biomechanical modeling.

\section{B. Related Work}

Buchanan et al. [1], Flanders and Soechting [2], Gomi [5] and van Bolhuis and Gielen [11] have studied isometric experiments, which focus on the muscle load-sharing freedoms

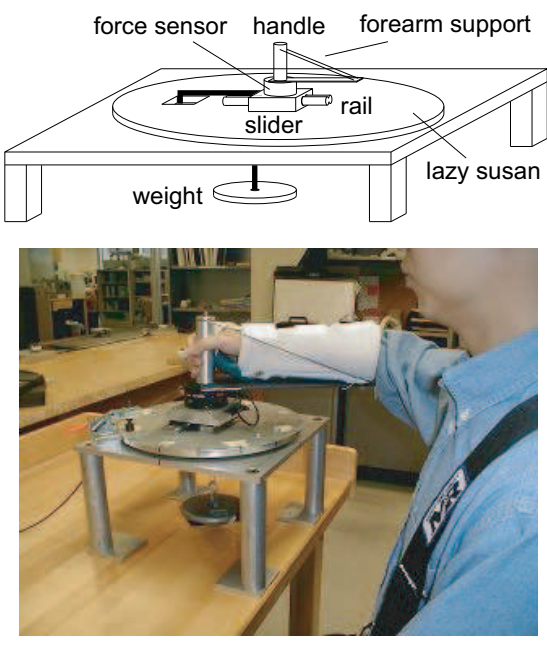

Fig. 2. Experimental setup.

(4) from the Introduction. On the other hand, a great deal of work has studied how the motion freedoms (1) are resolved in unconstrained reaching motions. With a biomechanical model, muscle load-sharing freedoms (4) can also be included in determining natural unconstrained reaching motion.

For human arm (shoulder and elbow) unconstrained pointto-point motions, two popular models are minimum Cartesian jerk at the hand (Flash and Hogan [3]), and minimum squared rate of change of torque at the shoulder and elbow joints (Uno, Kawato, and Suzuki [9]).

Gomi [4] showed that the natural stiffness at the hand during motion is altered in the presence of a guiding constraint. Other previous work on partially constrained arm motion includes work examining how subjects turn a crank (Russell and Hogan [7]; Svinin et al. [8]). Russell and Hogan showed that people apply significant radial forces (compressing or extending the crank) even though they are workless. Svinin et al. compared experimental crank rotation force and motion profiles to those predicted by a model minimizing a weighted combination of hand force change and joint torque change.

\section{EXPERIMENTS}

\section{A. Setup and protocol}

Subjects were seated in a custom-made high-backed chair and grasped a vertical handle on a slider on a horizontal low-friction linear rail. The rail is mounted on a lazy susan, allowing the rail to be rotated 360 degrees in the plane (Figure 2). A forearm support is attached to the handle. This support maintains the arm in a horizontal plane throughout the experiments without fatiguing the subject.

Each trial consisted of the subject holding the handle while a weight was hung from a cable attached to the slider, causing a tangential pulling force on the slider. The subject then stabilized the position of the handle at the center of the rail by sight. Forces normal to the rail were then recorded for one second and averaged. The weight was then removed from the cable. 
In most experiments, the slider handle was located at $(0,45 \mathrm{~cm})$ in a frame fixed to the shoulder (Figure 10). (In Section IV we discuss different arm configurations.) Sixteen angles of the rail were used, evenly spaced at $22.5^{\circ}$ intervals around the unit circle. At each of the sixteen test positions, two different weights are hung from the cable, $0.858 \mathrm{~kg}$ (the light weight) and $1.759 \mathrm{~kg}$ (the heavy weight). These result in tangential forces of $8.4 \mathrm{~N}$ and $17.3 \mathrm{~N}$, respectively. For each angle and weight, the experiment was repeated three times. Therefore, for each subject, we collected $16 \times 2 \times 3=96$ data points.

\section{B. Experimental Results}

The measured force applied by a subject in a given trial can be decomposed into two orthogonal components: a component tangential to the rail denoted $\mathbf{f}_{t}$, and whose value is predictably equal to the pulling force, and a component perpendicular to the rail denoted $\mathbf{f}_{n}$, which is the object of study.

We recruited eight healthy right-handed male subjects for our experiments. The experimental results of one subject are shown in Figures 3 as plots of the applied normal force $f_{n}$ as a function of the angle of the tangential force applied by the subject (the $+f_{t}$-axis). Two plots are shown: one for the light weight and one for the heavy weight. For each plot, the dotted line shows the average applied constraint force over the three trials. The shaded region shows the range of normal forces measured over the three trials. The solid line is identical to the dotted line of average normal forces, except it has been shifted up or down so that its integral over all test angles is zero (zero mean), a consideration that will be discussed later.

Simple observation of the data indicates that subjects often apply force normal to the constraint, depending on the angle of the constraint and the direction of the tangent force, even though normal forces are not necessary for the task. In fact, for several of the subjects, the peak value of the normal force is about as large as the required force along the rail. For the most part, inter-trial variations are small relative to the peak constraint forces.

\section{Force Scaling}

We would like to know if subjects' normal force $f_{n}$ scales approximately proportionally with the tangent force $f_{t}$ for a fixed tangent force direction. The scaling hypothesis can be written

$$
\frac{8.4}{17.3} f_{n, \text { heavy }}-f_{n, \text { light }}=z=0,
$$

where $f_{n, h e a v y}$ and $f_{n, l i g h t}$ are the normal forces applied by a given subject at a given angle of the tangent force $f_{t}$ for the large $(17.3 \mathrm{~N})$ and small $(8.4 \mathrm{~N})$ tangential forces, respectively. First we performed a two-way ANOVA with "constraint angle" and "force magnitude" as experimental factors when subject data were pooled. It showed no main effect for "force magnitude" factor on the data $(\mathrm{F}=1.143$, $\mathrm{p}=0.286$ ). Also there was no evidence of system deviation from scaling hypothesis across subjects for "constraint angle $\times$ force magnitude" interaction $(\mathrm{F}=0.586, \mathrm{p}=0.884$, adjusted

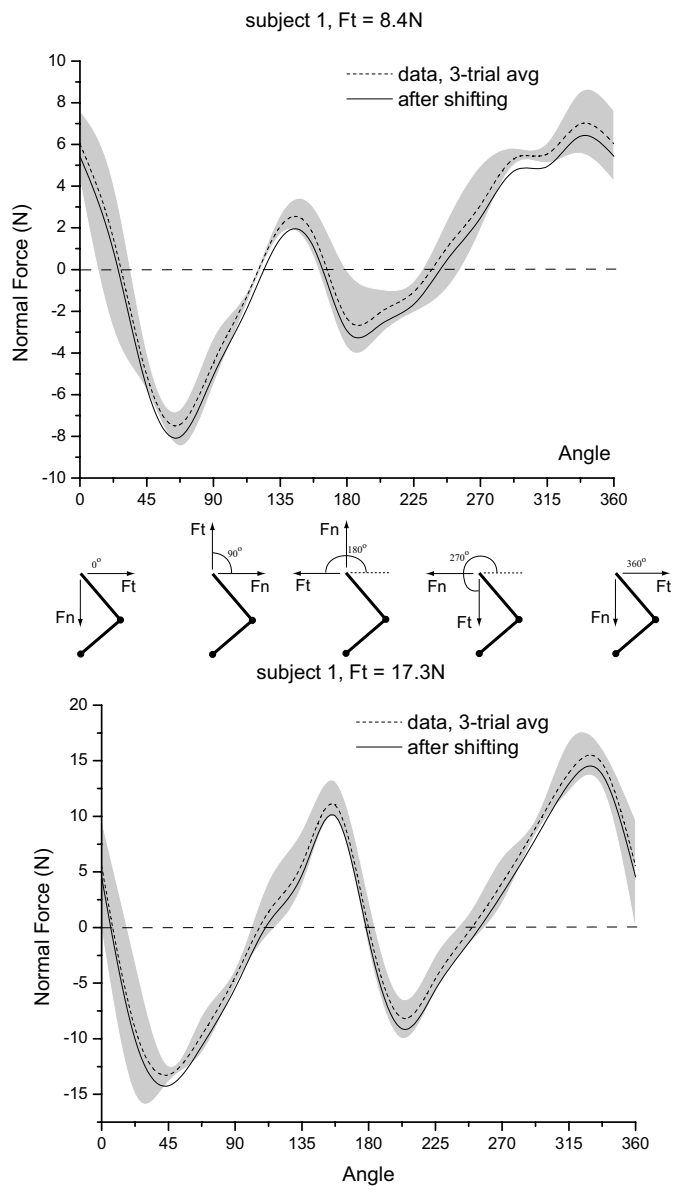

Fig. 3. Subject 1 normal force plot.

$R^{2}=0.62$ ). Then we performed two-way ANOVAs for each subject $(n=8)$. They showed no main effect for the "force magnitude" factor except for one subject. The results also showed that there are some idiosyncratic deviations from the scaling hypothesis within each subject due to the "constraint angle $\times$ force magnitude" interaction. However, the pooled analysis we did before already showed that deviations from the scaling hypothesis were different for different subjects and tended to cancel out or be obscured by the inter-subject variability. Other analysis, like correlation coefficients for linear fit $(0.876 \pm 0.053)$, also indicate that scaling hypothesis is reasonable.

\section{ObJective Functions And Iso-Cost Contours}

We would like to find an organizing principle to predict normal force data such as that found in Figures 3. This organizing principle can be expressed as an objective function that describes the "cost" to the subject of generating a particular force vector, or it may reflect some facet of the organization of the motor control system. In either case, the role of the objective function is to resolve the freedom in the applied constraint force.

The objective function $g\left(\mathbf{f}_{h}\right)$ can be viewed as a function 


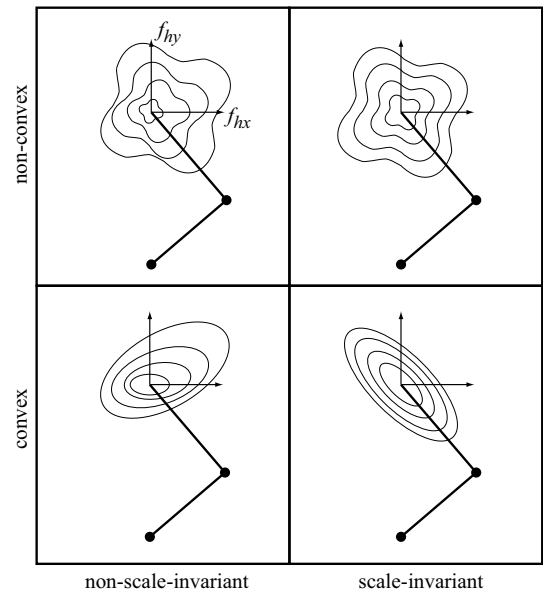

Fig. 4. Different classes of objective functions as represented by their iso-cost contours. Top left: A general bowl-shaped objective function. Top right: A non-convex scale-invariant objective function. Bottom left: A convex non-scale-invariant objective function. Bottom right: A convex scale-invariant objective function.

on the hand force space $g: \mathbb{R}^{2} \rightarrow \mathbb{R}$, where $g$ gives the "cost" of the hand force $\left(f_{h x}, f_{h y}\right)$. Thus the objective function forms a two-dimensional surface. A weak assumption on the form of $g$ is that the cost increases monotonically as we move outward along any ray from the origin. For low to moderate hand forces, this seems intuitively correct: the "cost" for a nonzero hand force $\mathbf{f}_{h}$ is greater than for any hand force $\alpha \mathbf{f}_{h}$ for $0 \leq \alpha<1$. An objective function satisfying this condition defines a bowl in the force space, and there are no local minima except the global minimum at $(0,0)$. The level curves of a bowl-shaped cost function are concentric, closed, and star-shaped about the origin. We will call these level curves iso-cost contours in the hand force space.

Two interesting subclasses of bowl-shaped objective functions are those whose iso-cost contours are all convex, and those whose iso-cost contours are scale-invariant - each isocost contour is a uniformly-scaled version of every other. These properties are shown graphically in Figure 4. The Hessian matrix $\nabla_{\mathbf{f}_{h} \mathbf{f}_{h}}^{2} g$ of a convex objective function is positive definite at all $\mathbf{f}_{h}$, i.e.,

$$
\begin{aligned}
\operatorname{trace}\left(\nabla_{\mathbf{f}_{h} \mathbf{f}_{h}}^{2} g\right) & >0 \\
\operatorname{det}\left(\nabla_{\mathbf{f}_{h} \mathbf{f}_{h}}^{2} g\right) & >0 .
\end{aligned}
$$

Scale-invariant objective functions satisfy the property

$$
g\left(\alpha \mathbf{f}_{h}\right)=k(\alpha) g\left(\mathbf{f}_{h}\right), \quad \alpha, k(\alpha) \in \mathbb{R}^{+},
$$

for some scaling $k(\alpha)$ satisfying $k(1)=1$ and monotonically increasing with $\alpha$. A common example of $k(\alpha)$ is a power law $\alpha^{p}$. If an objective function is both convex and scale-invariant, we refer to it as a CSI objective function. These properties of an objective function generalize immediately to $\mathbf{f}_{h}$ in more than two dimensions.

How can an objective function be used to predict a constraint force? Consider the general case of an $n$ degree-offreedom load and $m$ constraints. The subject must apply a
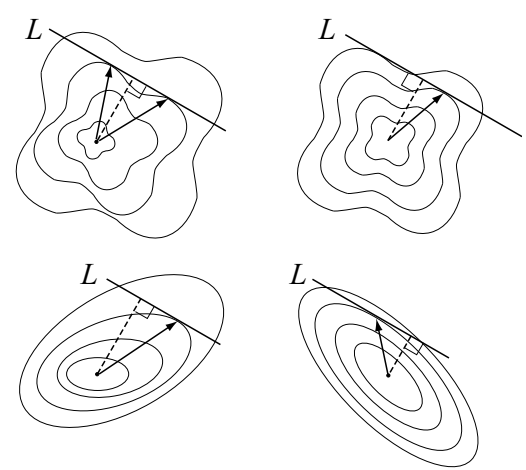

Fig. 5. In each figure, the dotted line represents $\mathbf{f}_{t}$, and the line $L$ is the line of equivalent forces along the rail. The arrows represent the optimal total force $\mathbf{f}_{h}=\mathbf{f}_{t}+\mathbf{f}_{n}$. The line $L$ in the top left figure is tangent to the same isocost contour at two distinct points, meaning that it achieves a cost minimum at two different $\mathbf{f}_{h}$. This is only possible with non-convex iso-cost contours. In all other figures a unique optimum is predicted.

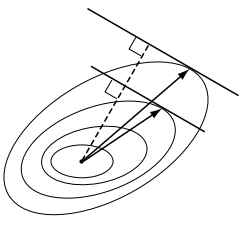

(a)

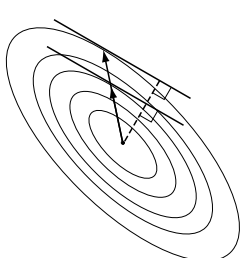

(b)
Fig. 6. (a) For some objective functions, scaling the force $\mathbf{f}_{t}$ (dotted lines) does not result in a scaled optimal hand force $\mathbf{f}_{h}$ (arrows). (b) For scaleinvariant objective functions, scaling the force $\mathbf{f}_{t}$ proportionally scales the optimal hand force $\mathbf{f}_{h}$.

specific force $\mathbf{f}_{t}$ to satisfy the static task requirements. The subject is free to apply any normal force $\mathbf{f}_{n}$ without affecting the task. Therefore, there is an $m$-dimensional linear subspace of generalized forces containing $\mathbf{f}_{t}$ that satisfies the task. The objective function predicts that the subject will choose the point in this linear subspace that minimizes the cost. At this point, the linear subspace will be tangent to one of the $(n-1)$ dimensional iso-cost surfaces. (If the cost function is nonconvex, a tangent point is not necessarily a minimum.)

For our particular example, $m=1$ and the linear subspace is simply a line. Prediction of the normal force can be done by a graphical construction. On the plot of iso-cost contours, construct the line $L$ passing through the point $\mathbf{f}_{t}=\left(f_{t x}, f_{t y}\right)$ and perpendicular to the direction of the rail. This is the linear subspace of forces satisfying the task. The optimal total force $\mathbf{f}_{h}=\mathbf{f}_{t}+\mathbf{f}_{n}$ occurs where the line is tangent to an iso-cost contour. Examples of the construction are shown in Figure 5.

If the objective function is scale-invariant, the direction of the optimal total vector force $\mathbf{f}_{h}$ depends only on the direction of $\mathbf{f}_{t}$, and not on its magnitude. In other words, if the required tangent force $\mathbf{f}_{t}$ is scaled by $\alpha$, then the optimal normal force is also scaled by $\alpha$. This does not hold for more general objective functions, as shown in Figure 6.

In Section II-C we statistically tested the possibility of scale- 
invariance, and we found that the data were consistent with the hypothesis. In addition, as we will see later, the fact that the experimental normal force plots (such as Figure 3) have approximately zero mean can be seen as further evidence of the scale-invariance of the objective function. Also, every minimization model for static muscle load-sharing we are aware of in the literature (muscle group stresses or tensions, or joint torques, raised to the power of 1,2 , or 3 and summed) implies a CSI (convex and scale-invariant) objective function at the hand. ${ }^{2}$ Several researchers have found that as the direction of an applied force remains fixed but the magnitude is scaled, the muscle activation and force patterns simply scale, further evidence of scale-invariance (Buchanan et al. [1]; Flanders and Soechting [2]; Valero-Cuevas et al. [10]). For these reasons, we will proceed with the assumption that the objective function is scale-invariant. This assumption will allow us to derive the shape of the iso-cost contours implied by the experimental data without any detailed biomechanical modeling or more restrictive conditions on the form of the objective function.

\section{A. Reconstructing Iso-Cost Contours}

The ability to reconstruct iso-cost contours is perhaps nonobvious, because the experiments do not keep the subject on the same iso-cost contour. In fact, there is no way to design the experiments to do so, since we do not know the iso-cost contours in advance. Since we are assuming scale-invariant objective functions, however, it is possible to derive the shape of an iso-cost contour (and therefore all iso-cost contours) from the experimental data. The reconstruction proceeds as follows.

Each point on a normal force plot indicates a point in the hand space $\left(f_{h x}, f_{h y}\right)$ space, at an angle $\beta$ relative to the $+f_{h x}$ axis. At this point, the direction of the normal force $f_{n}$ is tangent to the iso-cost contour, as shown in Figure 6(b). Therefore, the $p$ data points of the normal force plot gives us a set of angles $\beta_{i}, i=1 \ldots p$ and a tangent direction $\gamma_{i}$ associated with each $\beta_{i}$. The iso-cost contour must be generated from this data. Choosing a point at an arbitrary radius $r_{1}$ (say $r_{1}=1$ ) along a ray at angle $\beta_{1}$ from the origin of the $\left(f_{h x}, f_{h y}\right)$ space, integrate angularly using the tangent angle $\gamma_{1}$ until $\beta_{2}$ is reached. Then using angle $\gamma_{2}$, integrate until $\beta_{3}$ is reached, and so on. (More sophisticated interpolating numerical integration could instead be used.) Continue around angularly until the curve reaches $\beta_{1}$ again. If the normal force plot comes from a scale-invariant objective function, the curve will close at $\beta_{1}$. The key point is that for scale-invariant objective functions, the tangent direction $\gamma$ depends only on the angle $\beta$ of the force $\mathbf{f}_{h}$, not the magnitude $\left\|\mathbf{f}_{h}\right\|$.

\footnotetext{
${ }^{2}$ Any CSI objective function defined in the joint torque, muscle tension, or muscle stress space maps to a CSI objective function in the hand force space, as linear mappings (from muscle stress to muscle tension, from muscle tension to joint torques, and from joint torques to hand forces) preserve convexity and scale-invariance.
}

What if the curve does not close after integrating the tangents around 360 degrees? This can certainly happen because of numerical integration errors, but could there be a more fundamental reason? What condition does the normal force experimental data have to satisfy to result in a closed reconstructed iso-cost contour? It turns out that the integral of the normal force curve must equal zero, i.e., the normal forces must be zero mean.

Proposition 3.1: The experimental normal force curve must be zero mean to yield a closed scale-invariant iso-cost contour by the integration procedure outlined above.

\section{(Proof omitted for space.)}

To reconstruct the iso-cost contour from experimental data, we first shift the curve of average normal forces by subtracting the mean value of the normal force. This shifts the curve up or down by a fixed amount and creates a zero-mean curve. As can be seen from the plots in Figure 3, the amount of the shift is generally small relative to the amplitude of the maximum normal forces. Also, the shifted average curves remain almost everywhere inside the range of normal force values established by the three trials. Thus the shift is supported by the variations in the experimental data. This indicates that the actual experimental normal force data are indeed approximately zero mean, as needed to reconstruct the iso-cost contours.

The fact that the experimental normal force curves are zero mean (within trial-to-trial variations) can be seen as further evidence of a scale-invariant objective function. While all scale-invariant objective functions predict zero mean normal force curves, this is not true for general non-scale-invariant objective functions.

Four subjects' reconstructed iso-cost contours from the normal force data (after shifting) for the hand position $(0,45 \mathrm{~cm})$ in the shoulder frame are shown in Figure 7. Because the objective function is assumed scale-invariant, only the shape of the iso-cost contours is of interest; their sizes are arbitrary.

We can make a few general observations about the shapes of the iso-cost contours. Almost all of the reconstructed contours are convex, implying a convex objective function. For most subjects, the shapes of the iso-cost contours found from the normal force data are almost the same for $\left|f_{t}\right|=8.4 \mathrm{~N}$ and $17.3 \mathrm{~N}$ in the experiments. This is predicted by scaleinvariance. The iso-cost contours are stretched in the $f_{h y}$ direction relative to the $f_{h x}$ direction, indicating that a larger force in the $f_{h y}$ direction has the same "cost" as a smaller force in the $f_{h x}$ direction. This is not surprising for this configuration of the arm, and similar stretching has been observed in experimentally derived impedance ellipses for the arm (e.g., Hogan [6]; Gomi [4]).

The key points of the reconstructed iso-cost contours are that $(i)$ they are found independent of any strong biomechanical modeling assumptions, and ( $i i)$ they represent how the constraint force freedoms (3) described in the Introduction are used by subjects in solving static manipulation tasks. 


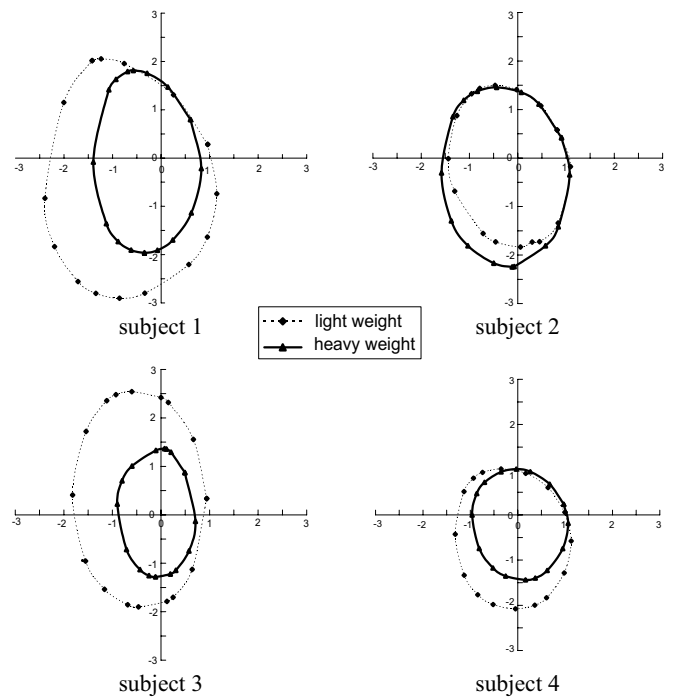

Fig. 7. The reconstructed iso-cost contours for four subjects at the hand position $(0,45 \mathrm{~cm})$ in the shoulder frame. The scale is insignificant; only the shape matters.

\section{B. Iso-cost contours predicted by biomechanical models}

Based on a simplified six muscle groups arm model (van Bolhuis and Gielen [11]; Gomi [5]) and physiological parameters of the muscle groups (Gomi [5]), we can use our data to distinguish how well different biomechanical models fit experimental data for natural interaction with a constraint. We will consider the following minimization models for interaction with the constraint:

- HAND Hand force magnitude $\|\mathbf{f}\|$ is minimized. According to this model, the subject applies only forces tangent to the rail. The constraint force is zero.

- T2 Torque squared, $\sum_{i} \tau_{i}^{2}$. For a robot arm with identical DC motors at the shoulder and elbow, this solution minimizes the electrical power to the motors.

- MT1 Muscle tension, $\sum_{i} \phi_{i}, i \in\{s e, s f, e e, e f$, $b e, b f\}$ represents shoulder extensor and flexor, elbow extensor and flexor, and biarticular extensor and flexor.

- MT2 Muscle tension squared, $\sum_{i} \phi_{i}^{2}$.

- MT3 Muscle tension cubed, $\sum_{i} \phi_{i}^{3}$.

- MS1 Muscle stress, $\sum_{i} \phi_{i} / P C S A_{i}$, where $P C S A_{i}$ is the physiological cross-sectional area of muscle $i$. This is a measure of the activation of the muscle.

- MS2 Muscle stress squared, $\sum_{i}\left(\phi_{i} / P C S A_{i}\right)^{2}$.

- MS3 Muscle stress cubed, $\sum_{i}\left(\phi_{i} / P C S A_{i}\right)^{3}$.

The linear models MT1 and MS1 tend to predict activation of only one of the muscle groups for a given task, while higherorder models predict greater sharing of the load across the muscle groups. Any model other than HAND indicates that the subject chooses a strategy with nonzero constraint forces.

Each of the eight models defines a CSI objective function in the hand force frame for a given arm configuration. For the T2 model, the iso-cost contours are ellipses that can be found in closed form. The iso-cost contours for the HAND model are

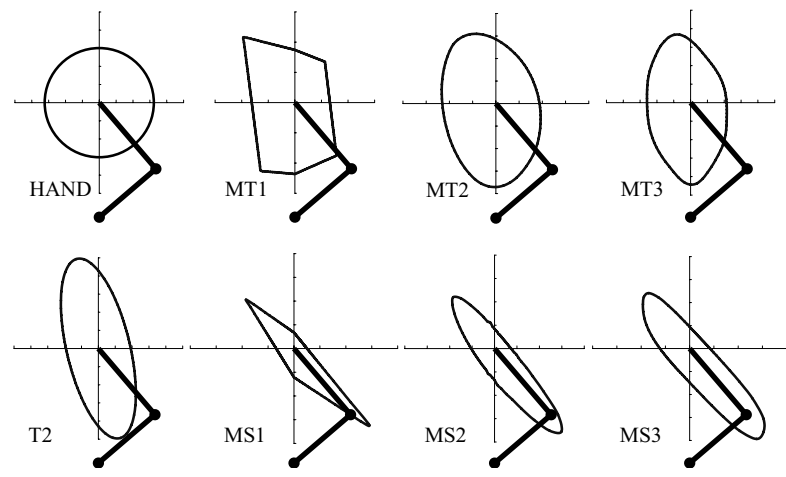

Fig. 8. The eight models of force generation imply iso-cost contours at the hand position $(0,45 \mathrm{~cm})$ in the shoulder frame with $L_{1}=30 \mathrm{~cm}, L_{2}=$ $35 \mathrm{~cm}$.

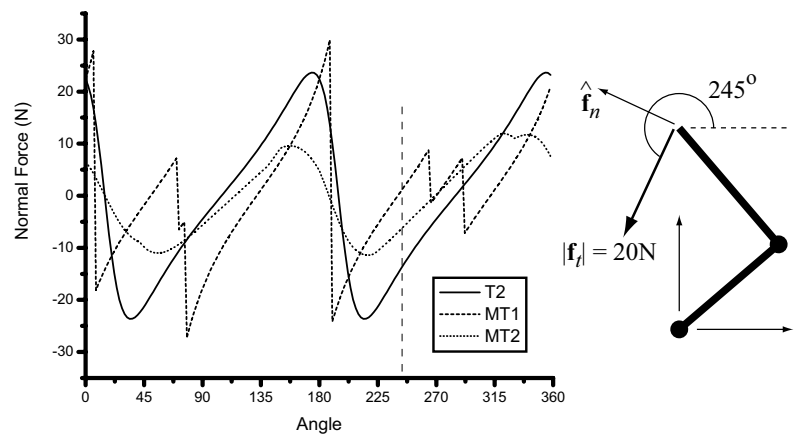

Fig. 9. The normal force predicted by three different models, T2, MT1, and MT2. The magnitude of the tangential force $\mathbf{f}_{t}$ (along the rail) applied by the human is $20 \mathrm{~N}$, and the direction of $\mathbf{f}_{t}$ is varied between $0^{\circ}$ and $360^{\circ}$. The positive direction of the normal force is defined as $90^{\circ}$ clockwise of the direction of the tangential force, indicated by the vector $\hat{\mathbf{f}}_{n}$ in the figure. The link lengths of the arm are $L_{1}=30 \mathrm{~cm}, L_{2}=35 \mathrm{~cm}$ and the arm is at the $(0,45 \mathrm{~cm})$ hand position. The figure shows a tangential force at $245^{\circ}$, and the three models predict different normal forces.

simply circles centered at the origin. Iso-cost contours for the other models can be found numerically (Figure 8). The linear models MT1 and MS1 result in polygonal iso-cost contours; the other models have strictly convex iso-cost contours. Note the strong anisotropy of the MS $k$ iso-cost contours, due to the presumed large PCSA of the uniarticular shoulder muscles.

Each of the models predicts a normal force plot that can be compared to a subject's experimental data, as in Figure 3. Figure 9 shows predicted normal force plots for three different models. Each radial local maximum and minimum of the isocost contour predicts zero normal force. For the HAND model, all points of an iso-cost contour are extremal, and it always predicts zero normal force.

\section{DisCUSSION AND FUtURE WORK}

The results clearly show that subjects make non-trivial use of the kinematic constraints, suggesting that passive guides may be useful to a human manipulating a heavy load. This can be seen from the anisotropy of subjects' reconstructed iso-cost contours. Although the iso-cost contours are anisotropic, they 


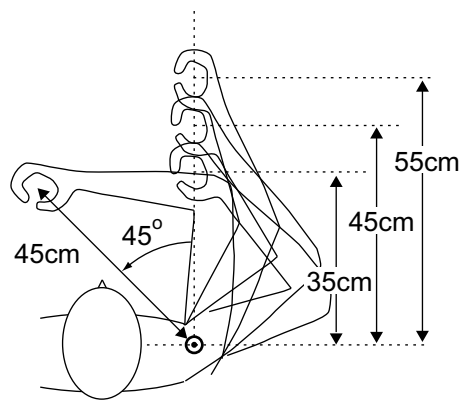

Fig. 10. Other test hand positions included the original point rotated $45^{\circ}$ in the shoulder frame, as well as the points $(0,35 \mathrm{~cm})$ and $(0,55 \mathrm{~cm})$.

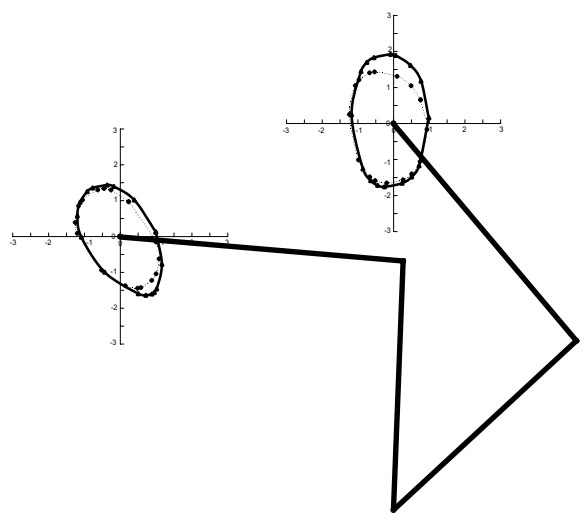

Fig. 11. Reconstructed iso-cost contours for subject 7 at the original test point and LEFT.

are not as anisotropic as those for the robotic T2 model. This suggests that biarticular muscles may act to make force properties at the hand more isotropic, as suggested by Hogan [6].

In addition to our tests at the hand position $(0,45 \mathrm{~cm})$, we performed the same experiments with a subset of subjects at hand positions $(0,55 \mathrm{~cm})$ (labeled FAR), $(0,35 \mathrm{~cm})$ (labeled NEAR), and $(-31.8 \mathrm{~cm}, 31.8 \mathrm{~cm})$ (labeled LEFT) in the shoulder frame. LEFT is the initial test position rotated $45^{\circ}$ in the shoulder frame (Figure 10).

Each of the models in Section III-B predicts that the isocost contour at LEFT has the same shape as the iso-cost contour at the original position, rotated $45^{\circ}$. This is in fact what we see in the reconstructed iso-cost contours for subject 7 (Figure 11). Because the elbow is more extended at the FAR position, each model predicts that the iso-cost contour becomes more anisotropic (stretched). This is what we see for subject 8 (Figure 12).

Our next set of experiments will study constrained pointto-point motion for the arm in the plane. We have built a planar 2R manipulandum capable of implementing smooth programmable guiding paths of arbitrary shape [12]. The manipulandum relies on a steered unicycle wheel at its endpoint to direct the motion over a plane. These experiments will allow us to study dynamic models of interaction with a constraint. We hypothesize that, as with the static examples presented

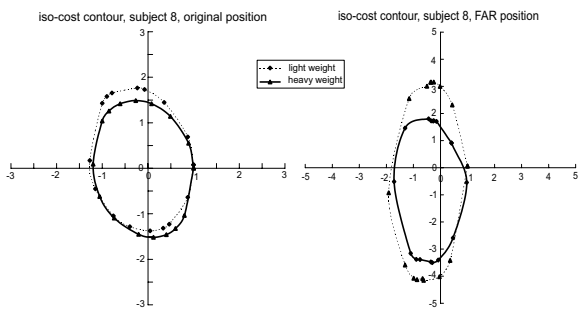

Fig. 12. Reconstructed iso-cost contours for subject 8 at the original test point and FAR.

above, even practiced interaction will involve considerable normal forces. These normal forces may allow us to distinguish between different dynamic models that predict similar trajectories in the unconstrained case. An open question is whether a single principle can be used to describe practiced forces and motions in unconstrained, partially constrained, and fully constrained manipulation tasks.

\section{Acknowledgments}

This work was funded by NSF grants IIS-9875469 and IIS0082957.

\section{REFERENCES}

[1] T. S. Buchanan, D. P. Almdale, J. L. Lewis, and W. Z. Rymer. Characteristics of synergic relations during isometric contractions of human elbow muscles. Journal of Neurophysiology, 56(5):1225-1241, 1986.

[2] M. Flanders and J. Soechting. Arm muscle activation for static forces in three-dimensional space. Journal of Neurophysiology, 64(6):1818-1837, 1990.

[3] T. Flash and N. Hogan. The coordination of arm movements: An experimentally confirmed mathematical model. The Journal of Neuroscience, 5:1688-1703, 1985

[4] H. Gomi. Anisotropic stiffness reduction during constrained multijoint arm movement. In Proceedings of the 20th Annual International Conference of the IEEE Engineering in Medicine and Biology Society, volume 20, pages 2336-2337, 1998.

[5] H. Gomi. Minimum principles for muscle-coordination evaluated by the directional preference of muscle activation during multijoint-arm force regulation tasks. Technical report, NTT Communication Science Labs, 2000 .

[6] N. Hogan. Adaptive control of mechanical impedance by coactivation of antagonist muscles. IEEE Transactions on Automatic Control, 29(8):681-690, Aug. 1984.

[7] D. Russell and N. Hogan. Dealing with constraints: A biomechanical approach. In Proc. IEEE Conference on Engineering in Medicine and Biology, 1989.

[8] M. Svinin, K. Ohta, Z. W. Luo, and S. Hosoe. Towards understanding of human movements constrained by the external environment. In IEEE/RSJ International Conference on Intelligent Robots and Systems, pages 155-161, 2003.

[9] Y. Uno, M. Kawato, and R. Suzuki. Formation and control of optimal trajectory in human multijoint arm movement: Minimum torque change model. Biological Cybernetics, 61:89-101, 1989.

[10] F. J. Valero-Cuevas, J. D. Towles, and V. R. Hentz. Quantification of fingertip force reduction in the forefinger following simulated paralysis of extensor and intrinsic muscles. Journal of Biomechanics, 33(12):1601$1609,2000$.

[11] B. M. van Bolhuis and C. Gielen. A comparison of models explaining muscle activation patterns for isometric contractions. Biological Cybernetics, 81:249-261, 1999.

[12] T. Worsnopp, M. Peshkin, E. Colgate, and K. Lynch. Controlling the apparent inertia of passive human interactive robots. In Proceedings of IEEE International Conference on Robotics and Automation, 2004. 